Annals of Pure and Applied Mathematics

Vol. 14, No. 3, 2017, 407-415

ISSN: 2279-087X (P), 2279-0888(online)

Published on 13 October 2017

www.researchmathsci.org

DOI: http://dx.doi.org/10.22457/apam.v14n3a7

Annals of

Pure and Applied

Mathematics

\title{
Fixed Point Theorem and Semi-Compatibility in Menger Probabilistic Metric Space
}

\author{
V.H.Badshah ${ }^{1}$, Suman Jain ${ }^{2}$ and Subhash Mandloi ${ }^{1}$ \\ ${ }^{1}$ School of Studies in Mathematics, Vikram University, Ujjain-456010 \\ Madhya Pradesh, India. \\ ${ }^{2}$ Department of Mathematics, Govt. College, Kalapipal (M.P.) India. \\ ${ }^{2}$ Corresponding author. Email: arihant2412@ gmail.com
}

Received 2 September 2017; accepted 2 October 2017

Abstract. The present paper deals with a fixed point theorem for six self maps using the concept of semi-compatible self maps in a Menger PM-space. Our result generalizes the result of Singh and Sharma [12].

Keywords: Common fixed points, compatible maps, semi-compatible maps, t-norm.

AMS Mathematics Subject Classification (2010): 47H10, 54H25

\section{Introduction}

There have been a number of generalizations of metric space. One such generalization is Menger space initiated by Menger [7]. It is a probabilistic generalization in which we assign to any two points $x$ and $y$, a distribution function $F_{x, y}$. Schweizer and Sklar [9] studied this concept and gave some fundamental results on this space. Sehgal and Bharucha-Reid [10] extended the notion of contraction mapping to the setting of the Menger space. They obtained a generalization of the classical Banach contraction principle on complete Menger spaces.

The notion of compatible mapping in a Menger space has been introduced by Mishra [8]. Singh and Sharma [12] have proved a common fixed point theorem for four compatible maps in Menger space by taking a new inequality. Using the concept of compatible mappings of type (A) and weak compatible mappings, Jain et al. [2, 3, 4] proved some interesting fixed point theorems in Menger space. Cho, Sharma and Sahu [1] introduced the concept of semi-compatibility in a d-complete topological space. In Menger space, Singh et al. [11] defined the concept of semi-compatibility of pair of selfmaps. Using the concept of occasionally weakly compatible mappings, Jha et. al. [5] proved fixed point theorems in semi-metric space. Afterwards, Jha et al. [6] proved a common fixed point theorem for reciprocal continuous compatible mappings in metric space. In the sequel, Srinivas et al. [13] gave Djoudi's common fixed point theorem on compatible mappings of type $(\mathrm{P})$.

In this paper, we generalize the result of Singh and Sharma [12] by introducing the notion of semi-compatible self maps. 
V.H.Badshah, Suman Jain and Subhash Mandloi

\section{Preliminaries}

Definition 2.1. [8] A mapping $\boldsymbol{F}: \mathrm{R} \rightarrow \mathrm{R}^{+}$is called a distribution if it is non-decreasing left continuous with $\inf \{\boldsymbol{F}(\mathrm{t}) \mid \mathrm{t} \in \mathrm{R}\}=0$ and $\sup \{\boldsymbol{F}(\mathrm{t}) \mid \mathrm{t} \in \mathrm{R}\}=1$.

We shall denote by $\mathrm{L}$ the set of all distribution functions while $\mathrm{H}$ will always denote the specific distribution function defined by

$$
\mathrm{H}(\mathrm{t})= \begin{cases}0, & \mathrm{t} \leq 0 \\ 1, & \mathrm{t}>0\end{cases}
$$

Definition 2.2. [8] A mapping $\mathrm{t}:[0,1] \times[0,1] \rightarrow[0,1]$ is called a $t$-norm if it satisfies the following conditions :

$$
\begin{aligned}
& \mathrm{t}(\mathrm{a}, 1)=\mathrm{a}, \mathrm{t}(0,0)=0 ; \\
& \mathrm{t}(\mathrm{a}, \mathrm{b})=\mathrm{t}(\mathrm{b}, \mathrm{a}) ; \\
& \mathrm{t}(\mathrm{c}, \mathrm{d}) \geq \mathrm{t}(\mathrm{a}, \mathrm{b}) ; \text { for } \mathrm{c} \geq \mathrm{a}, \mathrm{d} \geq \mathrm{b}, \\
& \mathrm{t}(\mathrm{t}(\mathrm{a}, \mathrm{b}), \mathrm{c})=\mathrm{t}(\mathrm{a}, \mathrm{t}(\mathrm{b}, \mathrm{c})) .
\end{aligned}
$$

Definition 2.3. [8] A probabilistic metric space (PM-space) is an ordered pair (X, F) consisting of a non empty set $\mathrm{X}$ and a function $F: \mathrm{X} \times \mathrm{X} \rightarrow \mathrm{L}$, where $\mathrm{L}$ is the collection of all distribution functions and the value of $F$ at $(\mathrm{u}, \mathrm{v}) \in \mathrm{X} \times \mathrm{X}$ is represented by $\mathrm{F}_{\mathrm{u}, \mathrm{v}}$. The function $\mathrm{F}_{\mathrm{u}, \mathrm{v}}$ assumed to satisfy the following conditions:

$(\mathrm{PM}-1) \mathrm{F}_{\mathrm{u}, \mathrm{v}}(\mathrm{x})=1$, for all $\mathrm{x}>0$, if and only if $\mathrm{u}=\mathrm{v}$;

$(\mathrm{PM}-2) \mathrm{F}_{\mathrm{u}, \mathrm{v}}(0)=0$;

(PM-3) $\mathrm{F}_{\mathrm{u}, \mathrm{v}}=\mathrm{F}_{\mathrm{v}, \mathrm{u}}$;

(PM-4) If $\mathrm{F}_{\mathrm{u}, \mathrm{v}}(\mathrm{x})=1$ and $\mathrm{F}_{\mathrm{v}, \mathrm{w}}(\mathrm{y})=1$ then $\mathrm{F}_{\mathrm{u}, \mathrm{w}}(\mathrm{x}+\mathrm{y})=1$,

for all $\mathrm{u}, \mathrm{v}, \mathrm{w} \in \mathrm{X}$ and $\mathrm{x}, \mathrm{y}>0$.

A Menger space is a triplet $(\mathrm{X}, \mathcal{F}, \mathrm{t})$ where $(\mathrm{X}, \mathcal{F})$ is a PM-space and $\mathrm{t}$ is a t-norm such that the inequality

(PM-5) $\mathrm{F}_{\mathrm{u}, \mathrm{w}}(\mathrm{x}+\mathrm{y}) \geq \mathrm{t}\left\{\mathrm{F}_{\mathrm{u}, \mathrm{v}}(\mathrm{x}), \mathrm{F}_{\mathrm{v}, \mathrm{w}}(\mathrm{y})\right\}$, for all $\mathrm{u}, \mathrm{v}, \mathrm{w} \in \mathrm{X}, \mathrm{x}, \mathrm{y} \geq 0$.

Definition 2.4. [8] A sequence $\left\{\mathrm{x}_{\mathrm{n}}\right\}$ in a Menger space $(\mathrm{X}, \boldsymbol{F}, \mathrm{t})$ is said to be convergent and converges to a point $\mathrm{x}$ in $\mathrm{X}$ if and only if for each $\varepsilon>0$ and $\lambda>0$, there is an integer $\mathrm{M}(\varepsilon, \lambda)$ such that

$$
\mathrm{F}_{\mathrm{x}_{\mathrm{n}}, \mathrm{x}}(\varepsilon)>1-\lambda \quad \text { for all } \mathrm{n} \geq \mathrm{M}(\varepsilon, \lambda) .
$$

Further, the sequence $\left\{x_{n}\right\}$ is said to be Cauchy sequence if for $\varepsilon>0$ and $\lambda>0$, there is an integer $\mathrm{M}(\varepsilon, \lambda)$ such that

$$
\mathrm{F}_{\mathrm{x}_{\mathrm{n}}, \mathrm{x}_{\mathrm{m}}}(\varepsilon)>1-\lambda \quad \text { for all } \mathrm{m}, \mathrm{n} \geq \mathrm{M}(\varepsilon, \lambda) \text {. }
$$

A Menger PM-space $(\mathrm{X}, \boldsymbol{F}, \mathrm{t})$ is said to be complete if every Cauchy sequence in $\mathrm{X}$ converges to a point in $\mathrm{X}$. 
Fixed Point Theorem and Semi-Compatibility in Menger Probabilistic Metric Space

Definition 2.5. [8] Self maps $S$ and $T$ of a Menger space $(X, F, t)$ are said to be compatible if $\mathrm{F}_{\mathrm{STx}_{\mathrm{n}}}, \mathrm{TSx}_{\mathrm{n}}(\mathrm{x}) \rightarrow 1$ for all $\mathrm{x}>0$, whenever $\left\{\mathrm{x}_{\mathrm{n}}\right\}$ is a sequence in $\mathrm{X}$ such that $\mathrm{Sx}_{\mathrm{n}}, \mathrm{Tx}_{\mathrm{n}} \rightarrow \mathrm{u}$ for some $\mathrm{u}$ in $\mathrm{X}$, as $\mathrm{n} \rightarrow \infty$.

Definition 2.6. [11] Self maps $S$ and $T$ of a Menger space $(X, F, t)$ are said to be semi-

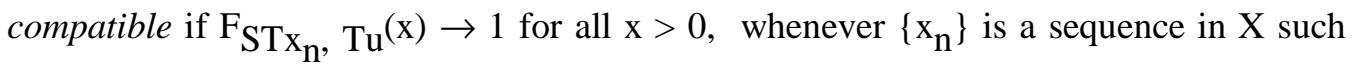
that $\mathrm{Sx}_{\mathrm{n}}, \mathrm{Tx}_{\mathrm{n}} \rightarrow \mathrm{u}$ for some $\mathrm{u}$ in $\mathrm{X}$, as $\mathrm{n} \rightarrow \infty$. STy $=$ TSy.

It follows that if the pair $(\mathrm{S}, \mathrm{T})$ is semi-compatible and $\mathrm{Sy}=\mathrm{Ty}$ then

Proposition 2.1. [11] If (S, T) is a semi-compatible pair of self maps in a Menger PMspace $(\mathrm{X}, \boldsymbol{F}, \mathrm{t})$ and $\mathrm{T}$ is continuous then $(\mathrm{S}, \mathrm{T})$ is compatible.

Proposition 2.2. [11] If $(X, d)$ is a metric space, then the metric $d$ induces a mapping $\mathrm{F}: \mathrm{X} \times \mathrm{X} \rightarrow \mathrm{L}$, defined by

$$
\mathrm{F}_{\mathrm{p}, \mathrm{q}}(\mathrm{x})=\mathrm{H}(\mathrm{x}-\mathrm{d}(\mathrm{p}, \mathrm{q})), \mathrm{p}, \mathrm{q} \in \mathrm{X} \text { and } \mathrm{x} \in \mathrm{R} .
$$

Further, if $\mathrm{t}:[0,1] \times[0,1] \rightarrow[0,1]$ is defined by $\mathrm{t}(\mathrm{a}, \mathrm{b})=\min \{\mathrm{a}, \mathrm{b}\}$, then $(\mathrm{X}, \boldsymbol{F}, \mathrm{t})$ is a Menger space. It is complete if $(X, d)$ is complete. The space $(X, F, t)$ is called an induced Menger space.

Remark 2.1. [11] The concept of semi-compatibility of pair of self maps is more general than that of compatibility.

Proposition 2.3. [8] If $S$ and $T$ are compatible self maps of a Menger space $(X, F, t)$ where $t$ is continuous and $t(x, x) \geq x$ for all $x \in[0,1]$ and $S x_{n}, T x_{n} \rightarrow u$ for some $u$ in $X$. Then $\mathrm{TSx}_{\mathrm{n}} \rightarrow \mathrm{Su}$ provided $\mathrm{S}$ is continuous.

Proposition 2.4. [4] Let $S$ and $T$ be compatible self maps of Menger space $(X, \mathcal{F}, \mathrm{t})$ and $\mathrm{Su}=\mathrm{Tu}$ for some $\mathrm{u}$ in $\mathrm{X}$ then $\mathrm{STu}=\mathrm{TSu}=\mathrm{SSu}=\mathrm{TTu}$.

Lemma 2.1. [4] Let $\left\{p_{n}\right\}$ be a sequence in a Menger space $(X, F, t)$ with continuous tnorm and $t(x, x) \geq x$. Suppose, for all $x \in[0,1]$, there exists $k \in(0,1)$ such that for all $\mathrm{x}>0$ and $\mathrm{n} \in \mathrm{N}$,

or

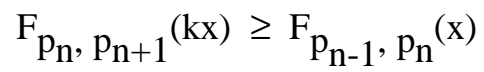

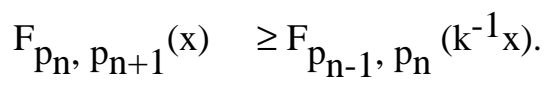

Then $\left\{p_{n}\right\}$ is a Cauchy sequence in $X$.

\section{Main results}

Theorem 3.1. Let A, B, S, T, L and $\mathrm{M}$ be self mappings of a complete Menger space (X, $\mathcal{F}, \mathrm{t})$ with $\mathrm{t}(\mathrm{a}, \mathrm{a}) \geq \mathrm{a}$, for some $\mathrm{a} \in[0,1]$, satisfying :
$\mathrm{L}(\mathrm{X}) \subseteq \mathrm{ST}(\mathrm{X}), \mathrm{M}(\mathrm{X}) \subseteq \mathrm{AB}(\mathrm{X}) ;$ 
V.H.Badshah, Suman Jain and Subhash Mandloi

$\mathrm{AB}=\mathrm{BA}, \quad \mathrm{ST}=\mathrm{TS}, \mathrm{LB}=\mathrm{BL}, \mathrm{MT}=\mathrm{TM}$;

either $\mathrm{AB}$ or $\mathrm{L}$ is continuous;

$(\mathrm{L}, \mathrm{AB})$ is compatible and $(\mathrm{M}, \mathrm{ST})$ is semi-compatible;

for all $\mathrm{p}, \mathrm{q} \in \mathrm{X}, \mathrm{x}>0$ and $0<\alpha<1$,

$$
\begin{aligned}
& {\left[\mathrm{F}_{\mathrm{Lp}, \mathrm{Mq}}(\mathrm{x})+\mathrm{F}_{\mathrm{ABp}, \mathrm{Lp}}(\mathrm{x})\right]\left[\mathrm{F}_{\mathrm{Lp}, \mathrm{Mq}}(\mathrm{x})+\mathrm{F}_{\mathrm{STq}}, \mathrm{Mq}^{(\mathrm{x})]}\right.}
\end{aligned}
$$

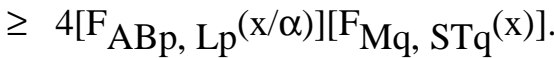

Then A, B, S, T, L and $\mathrm{M}$ have a unique common fixed point in $\mathrm{X}$.

Proof: Let $\mathrm{x}_{0} \in X$. From condition (3.1.5) $\exists \mathrm{x}_{1}, \mathrm{x}_{2} \in X$ such that

$$
\mathrm{Lx}_{0}=\mathrm{STx}_{1}=\mathrm{y}_{0} \text { and } \mathrm{Mx}_{1}=\mathrm{ABx}_{2}=\mathrm{y}_{1} \text {. }
$$

Inductively, we can construct sequences $\left\{x_{n}\right\}$ and $\left\{y_{n}\right\}$ in $X$ such that for $\mathrm{n}=0,1,2, \ldots$.

$$
\mathrm{Lx}_{2 \mathrm{n}}=\mathrm{STx}_{2 \mathrm{n}+1}=\mathrm{y}_{2 \mathrm{n}} \text { and } \mathrm{Mx}_{2 \mathrm{n}+1}=\mathrm{ABx}_{2 \mathrm{n}+2}=\mathrm{y}_{2 \mathrm{n}+1}
$$

Step 1. Putting $p=x_{2 n}, q=x_{2 n+1}$ for $x>0$ in (3.1.5), we get

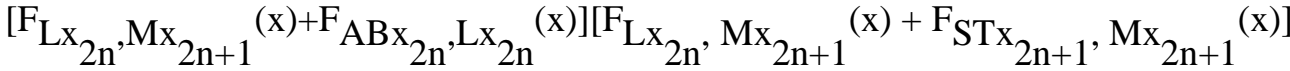

$$
\begin{aligned}
& \geq 4\left[\mathrm{~F}_{\mathrm{ABx}}{ }_{2 \mathrm{n}}, \mathrm{Lx}_{2 \mathrm{n}}(\mathrm{x} / \mathrm{a})\right]\left[\mathrm{F}_{\mathrm{Mx}_{2 \mathrm{n}+1}}, \mathrm{STx}_{2 \mathrm{n}+1}(\mathrm{x})\right]
\end{aligned}
$$

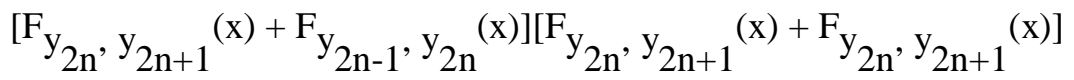

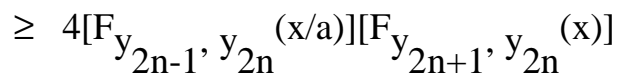

$$
\begin{aligned}
& \text { or, } \quad 2 \mathrm{~F}_{\mathrm{y}_{2 n}}, \mathrm{y}_{2 \mathrm{n}+1}(\mathrm{x})\left[\mathrm{F}_{\mathrm{y}_{2 n}}, \mathrm{y}_{2 \mathrm{n}+1}(\mathrm{x})+\mathrm{F}_{\mathrm{y}_{2 n-1}}, \mathrm{y}_{2 \mathrm{n}}(\mathrm{x})\right]
\end{aligned}
$$

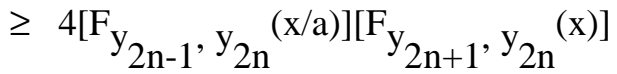

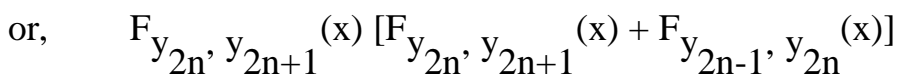

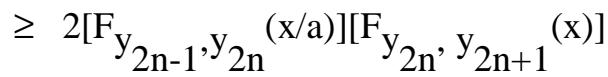

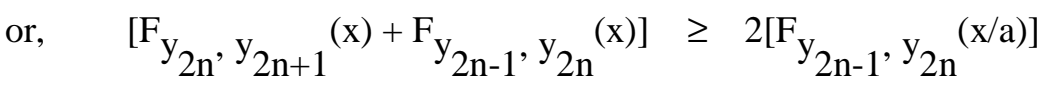

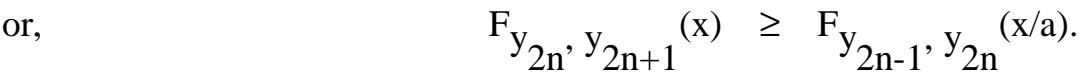

Similarly,

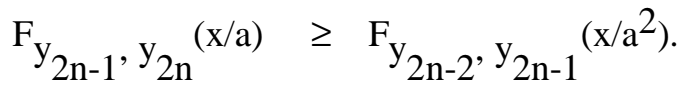

From (3.1.6) and (3.1.7), it follows that

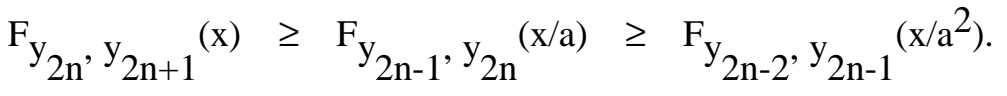

By repeated application of above inequality, we get

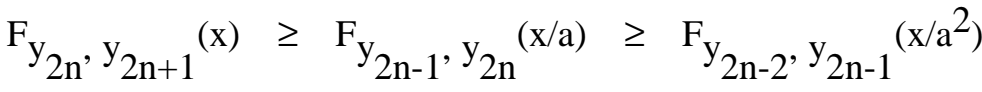

$$
\begin{aligned}
& \geq \ldots \geq \mathrm{F}_{\mathrm{y}_{0}, \mathrm{y}_{1}}\left(\mathrm{x} / \mathrm{a}^{\mathrm{n}}\right) \text {. }
\end{aligned}
$$


Fixed Point Theorem and Semi-Compatibility in Menger Probabilistic Metric Space

Therefore, by lemma 2.1, $\left\{\mathrm{y}_{\mathrm{n}}\right\}$ is a Cauchy sequence in $\mathrm{X}$, which is complete.

Hence $\left\{\mathrm{y}_{\mathrm{n}}\right\} \rightarrow \mathrm{z} \in \mathrm{X}$.

Also its subsequences converges as follows :

$$
\begin{aligned}
& \left\{\mathrm{Mx}_{2 \mathrm{n}+1}\right\} \rightarrow \mathrm{z} \\
& \left\{\mathrm{Lx}_{2 \mathrm{n}}\right\} \rightarrow \mathrm{z} \text { and }\left\{\mathrm{ABx}_{2 \mathrm{n}}\right\} \rightarrow \mathrm{z} .
\end{aligned}
$$

Case I. $\mathrm{AB}$ is continuous.

$\mathrm{As} \mathrm{AB}$ is continuous,

$$
(\mathrm{AB})^{2} \mathrm{x}_{2 \mathrm{n}} \rightarrow \mathrm{ABz} \text { and }(\mathrm{AB}) \mathrm{Lx}_{2 \mathrm{n}} \rightarrow \mathrm{ABz} .
$$

As $(\mathrm{L}, \mathrm{AB})$ is compatible, so by proposition (2.3), we have

$$
\mathrm{L}(\mathrm{AB}) \mathrm{x}_{2 \mathrm{n}} \rightarrow \mathrm{ABz} \text {. }
$$

Step 2. Putting $p=A B x_{2 n}$ and $q=x_{2 n+1}$ for $x>0$ in (3.1.5), we get

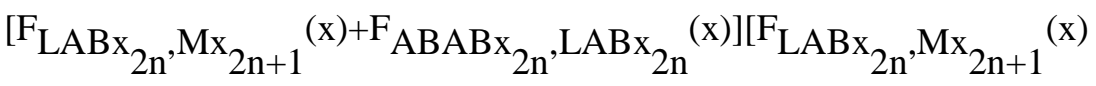

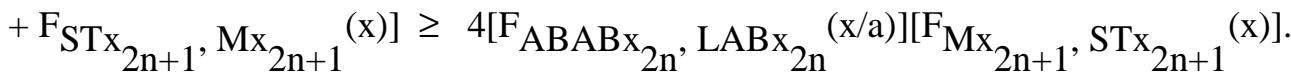

Letting $\mathrm{n} \rightarrow \infty$, we get

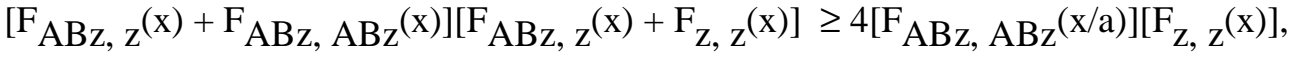

i.e. $\quad F_{A B z, z}(x) \geq 1$, yields $A B z=z$.

Step 3. Putting $p=z$ and $q=x_{2 n+1}$ for $x>0$ in (3.1.5), we get

$$
\begin{aligned}
& {\left[\mathrm{F}_{\mathrm{Lz}, \mathrm{Mx}} \mathrm{nn}_{\mathrm{n}+1}(\mathrm{x})+\mathrm{F}_{\mathrm{ABz}, \mathrm{Lz}}(\mathrm{x})\right]\left[\mathrm{F}_{\mathrm{Lz}}, \mathrm{Mx}_{2 \mathrm{n}+1}(\mathrm{x})+\mathrm{F}_{\mathrm{STx}} \mathrm{x}+1_{2 \mathrm{n}}, \mathrm{Mx}_{2 \mathrm{n}+1}(\mathrm{x})\right]} \\
& \geq 4\left[\mathrm{~F}_{\mathrm{ABz}, \mathrm{Lz}}(\mathrm{x} / \mathrm{a})\right]\left[\mathrm{F}_{\mathrm{Mx}_{2 \mathrm{n}+1}}, \mathrm{STx}_{2 \mathrm{n}+1}(\mathrm{x})\right] .
\end{aligned}
$$

Letting $\mathrm{n} \rightarrow \infty$, we get

$\left[\mathrm{F}_{\mathrm{Lz}, \mathrm{z}}(\mathrm{x})+\mathrm{F}_{\mathrm{z}, \mathrm{Lz}}(\mathrm{x})\right]\left[\mathrm{F}_{\mathrm{Lz}, \mathrm{z}}(\mathrm{x})+\mathrm{F}_{\mathrm{z}, \mathrm{z}}(\mathrm{x})\right] \geq 4\left[\mathrm{~F}_{\mathrm{z}, \mathrm{Lz}}(\mathrm{x} / \mathrm{a})\right]\left[\mathrm{F}_{\mathrm{z}, \mathrm{z}}(\mathrm{x})\right]$,

i.e. $\quad F_{L z},(x) \geq 1$, yields $\mathrm{Lz}=\mathrm{z}$.

Therefore, $\mathrm{ABz}=\mathrm{Lz}=\mathrm{z}$.

Step 4. Putting $p=B z$ and $q=x_{2 n+1}$ for $x>0$ in (3.1.5), we get

$$
\begin{aligned}
& {\left[\mathrm{F}_{\mathrm{LBz}}, \mathrm{Mx}_{2 \mathrm{n}+1}(\mathrm{x})+\mathrm{F}_{\mathrm{ABBz}, \mathrm{Bz}}(\mathrm{x})\right]\left[\mathrm{F}_{\mathrm{LBz}}, \mathrm{Mx}_{2 \mathrm{n}+1}(\mathrm{x})+\mathrm{F}_{\mathrm{STx}_{2 \mathrm{n}+1}, \mathrm{Mx}}{ }_{2 \mathrm{n}+1}(\mathrm{x})\right]} \\
& \geq 4\left[\mathrm{~F}_{\mathrm{ABBz}, \mathrm{LBz}}(\mathrm{x} / \mathrm{a})\right]\left[\mathrm{F}_{\mathrm{Mx}_{2 \mathrm{n}+1}}, \operatorname{STx}_{2 \mathrm{n}+1}(\mathrm{x})\right] .
\end{aligned}
$$

As $\mathrm{BL}=\mathrm{LB}, \mathrm{AB}=\mathrm{BA}$, so we have

$$
\mathrm{L}(\mathrm{Bz})=\mathrm{B}(\mathrm{Lz})=\mathrm{Bz} \text { and } \mathrm{AB}(\mathrm{Bz})=\mathrm{B}(\mathrm{ABz})=\mathrm{Bz} \text {. }
$$

Letting $\mathrm{n} \rightarrow \infty$, we get

$$
\left[\mathrm{F}_{\mathrm{Bz}, \mathrm{z}}(\mathrm{x})+\mathrm{F}_{\mathrm{Bz}, \mathrm{Bz}}(\mathrm{x})\right]\left[\mathrm{F}_{\mathrm{Bz}, \mathrm{z}}(\mathrm{x})+\mathrm{F}_{\mathrm{z}, \mathrm{z}}(\mathrm{x})\right] \geq 4\left[\mathrm{~F}_{\mathrm{Bz}, \mathrm{Bz}}(\mathrm{x} / \mathrm{a})\right]\left[\mathrm{F}_{\mathrm{z}, \mathrm{z}}(\mathrm{x})\right],
$$

i.e. $\quad F_{B z, z}(x) \geq 1$, yields $B z=z$ and $A B z=z$ implies $A z=z$.

Therefore, $\mathrm{Az}=\mathrm{Bz}=\mathrm{Lz}=\mathrm{z}$. 
V.H.Badshah, Suman Jain and Subhash Mandloi

Step 5. As $\mathrm{L}(X) \subseteq \mathrm{ST}(X)$, there exists $\mathrm{v} \in X$ such that $\mathrm{z}=\mathrm{Lz}=\mathrm{STV}$.

Putting $\mathrm{p}=\mathrm{x}_{2 \mathrm{n}}$ and $\mathrm{q}=\mathrm{v}$ for $\mathrm{x}>0$ in (3.1.5), we get

$$
\begin{aligned}
{\left[\mathrm{F}_{\left.\mathrm{Lx}_{2 n}, \mathrm{Mv}(\mathrm{x})+\mathrm{F}_{\mathrm{ABx}}{ }_{2 \mathrm{n}}, \mathrm{Lx}_{2 \mathrm{n}}(\mathrm{x})\right]\left[\mathrm{F}_{\mathrm{Lx}}, \mathrm{Mv}\right.}(\mathrm{x})+\mathrm{F}_{\mathrm{STv}, \mathrm{Mv}}(\mathrm{x})\right] } \\
\geq 4\left[\mathrm{~F}_{\mathrm{ABx}}{ }_{2 \mathrm{n}}, \mathrm{Lx}_{2 \mathrm{n}}(\mathrm{x} / \mathrm{a})\right]\left[\mathrm{F}_{\mathrm{Mv}}, \operatorname{STv}(\mathrm{x})\right] .
\end{aligned}
$$

Letting $\mathrm{n} \rightarrow \infty$ and using equation (3.1.9), we get

$\left[\mathrm{F}_{\mathrm{z}, \mathrm{Mv}}(\mathrm{x})+\mathrm{F}_{\mathrm{z}, \mathrm{z}}(\mathrm{x})\right]\left[\mathrm{F}_{\mathrm{z}, \mathrm{Mv}}(\mathrm{x})+\mathrm{F}_{\mathrm{z}, \mathrm{Mv}}(\mathrm{x})\right] \geq 4\left[\mathrm{~F}_{\mathrm{z}, \mathrm{z}}(\mathrm{x} / \mathrm{a})\right]\left[\mathrm{F}_{\mathrm{Mv}, \mathrm{z}}(\mathrm{x})\right]$,

i.e. $\quad \mathrm{F}_{\mathrm{z}, \mathrm{Mv}}(\mathrm{x}) \geq 1$, yields $\mathrm{Mv}=\mathrm{z}$.

Hence, $\mathrm{STv}=\mathrm{z}=\mathrm{Mv}$.

As (M, ST) semi-compatible, we have $\mathrm{STMv}=\mathrm{MST}$.

Thus, $\mathrm{STz}=\mathrm{Mz}$.

Step 6. Putting $\mathrm{p}=\mathrm{x}_{2 \mathrm{n}}, \mathrm{q}=\mathrm{z}$ for $\mathrm{x}>0$ in (3.1.5), we get

$$
\begin{aligned}
& {\left[\mathrm{F}_{\mathrm{Lx}_{2 \mathrm{n}}}, \mathrm{Mz}^{(\mathrm{x})+\mathrm{F}_{\mathrm{ABx}}}{ }_{2 \mathrm{n}}, \mathrm{Lx}_{2 \mathrm{n}}(\mathrm{x})\right]\left[\mathrm{F}_{\mathrm{Lx}}{ }_{2 \mathrm{n}}, \mathrm{Mz}^{(\mathrm{x})}+\mathrm{F}_{\mathrm{STz}, \mathrm{Mz}}(\mathrm{x})\right]} \\
& \geq 4\left[\mathrm{~F}_{\mathrm{ABx}}{ }_{2 \mathrm{n}}, \mathrm{Lx}_{2 \mathrm{n}}(\mathrm{x} / \mathrm{a})\right]\left[\mathrm{F}_{\mathrm{Mz}}, \mathrm{STz}^{(\mathrm{x})]} .\right.
\end{aligned}
$$

Letting $\mathrm{n} \rightarrow \infty$ and using equation (3.1.8) and Step 5, we get

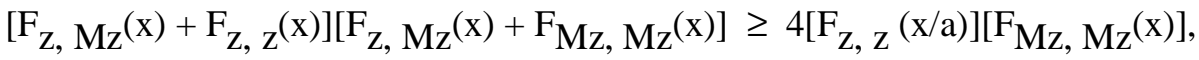
i.e. $\quad \mathrm{F}_{\mathrm{z}, \mathrm{Mz}}(\mathrm{x}) \geq 1$, yields $\mathrm{z}=\mathrm{Mz}$.

Step 7. Putting $\mathrm{p}=\mathrm{x}_{2 \mathrm{n}}$ and $\mathrm{q}=\mathrm{Tz}$ for $\mathrm{x}>0$ in (3.1.5), we get

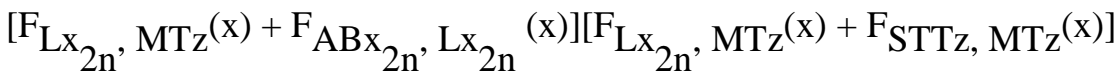

$$
\begin{aligned}
& \geq 4\left[\mathrm{~F}_{\mathrm{ABx}}{ }_{2 \mathrm{n}}, \mathrm{Lx}_{2 \mathrm{n}}(\mathrm{x} / \mathrm{a})\right]\left[\mathrm{F}_{\mathrm{MTz}}, \mathrm{STTz}^{(\mathrm{x})]}\right. \text {. }
\end{aligned}
$$

As MT $=\mathrm{TM}$ and $\mathrm{ST}=\mathrm{TS}$, we have $\mathrm{MTz}=\mathrm{TMz}=\mathrm{Tz}$ and $\mathrm{ST}(\mathrm{Tz})=\mathrm{T}(\mathrm{STz})=\mathrm{Tz}$. Letting $\mathrm{n} \rightarrow \infty$, we get

$\left[\mathrm{F}_{\mathrm{z}, \mathrm{Tz}}(\mathrm{x})+\mathrm{F}_{\mathrm{z}, \mathrm{z}}(\mathrm{x})\right]\left[\mathrm{F}_{\mathrm{z}, \mathrm{Tz}}(\mathrm{x})+\mathrm{F}_{\mathrm{Tz}, \mathrm{Tz}}(\mathrm{x})\right] \geq 4\left[\mathrm{~F}_{\mathrm{z}, \mathrm{z}}(\mathrm{x} / \mathrm{a})\right]\left[\mathrm{F}_{\mathrm{Tz}, \mathrm{Tz}}(\mathrm{x})\right]$,

i.e. $\quad \mathrm{F}_{\mathrm{z}, \mathrm{Tz}}(\mathrm{x}) \geq 1$, yields $\mathrm{Tz}=\mathrm{z}$.

Now $\quad \mathrm{STz}=\mathrm{Tz}=\mathrm{z}$ implies $\mathrm{Sz}=\mathrm{z}$.

Hence $\mathrm{Sz}=\mathrm{Tz}=\mathrm{Mz}=\mathrm{z}$.

Combining (3.1.11) and (3.1.12), we get

$$
\mathrm{Az}=\mathrm{Bz}=\mathrm{Lz}=\mathrm{Mz}=\mathrm{Tz}=\mathrm{Sz}=\mathrm{z} \text {. }
$$

Hence, the six self maps have a common fixed point in this case.

\section{Case II. $L$ is continuous}

As $\mathrm{L}$ is continuous, $\mathrm{L}^{2} \mathrm{x}_{2 \mathrm{n}} \rightarrow \mathrm{Lz}$ and $\mathrm{L}(\mathrm{AB}) \mathrm{x}_{2 \mathrm{n}} \rightarrow \mathrm{Lz}$.

As ( $\mathrm{L}, \mathrm{AB})$ is compatible, so by proposition (2.3), 
Fixed Point Theorem and Semi-Compatibility in Menger Probabilistic Metric Space

$$
(\mathrm{AB}) \mathrm{Lx}_{2 \mathrm{n}} \rightarrow \mathrm{Lz} \text {. }
$$

Step 8. Putting $p=\mathrm{Lx}_{2 n}$ and $q=x_{2 n+1}$ for $x>0$ in (3.1.5), we get

$$
\begin{aligned}
& {\left[\mathrm{F}_{\mathrm{LLx}_{2 \mathrm{n}}}, \mathrm{Mx}_{2 \mathrm{n}+1}(\mathrm{x})+\mathrm{F}_{\mathrm{ABLx}}{ }_{2 \mathrm{n}}, \operatorname{LLx}_{2 \mathrm{n}}(\mathrm{x})\right]\left[\mathrm{F}_{\mathrm{LLx}}{ }_{2 \mathrm{n}}, \mathrm{Mx}_{2 \mathrm{n}+1}(\mathrm{x})\right.} \\
& \left.+\mathrm{F}_{\mathrm{STx}} \mathrm{n}_{2 \mathrm{n}+1}, \mathrm{Mx}_{2 \mathrm{n}+1}(\mathrm{x})\right] \\
& \geq \quad 4\left[\mathrm{~F}_{\mathrm{ABLx}_{2 \mathrm{n}}}, \operatorname{LLx}_{2 \mathrm{n}}(\mathrm{x} / \mathrm{a})\right]\left[\mathrm{F}_{\mathrm{Mx}}{ }_{2 \mathrm{n}+1}, \mathrm{STx}_{2 \mathrm{n}+1}(\mathrm{x})\right] .
\end{aligned}
$$

Letting $\mathrm{n} \rightarrow \infty$, we get

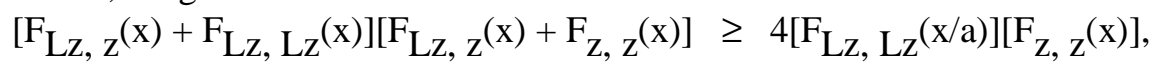

i.e. $\quad F_{\mathrm{Lz}, \mathrm{z}}(\mathrm{x}) \geq 1$, yields $\mathrm{Lz}=\mathrm{z}$.

Now, using steps 5-7, we get $\mathrm{Mz}=\mathrm{STz}=\mathrm{Sz}=\mathrm{Tz}=\mathrm{z}$.

Step 9. As $M(X) \subseteq A B(X)$, there exists $w \in X$ such that

$$
\mathrm{z}=\mathrm{Mz}=\mathrm{ABw} \text {. }
$$

Putting $\mathrm{p}=\mathrm{w}$ and $\mathrm{q}=\mathrm{x}_{2 \mathrm{n}+1}$ for $\mathrm{x}>0$ in (3.1.5), we get

$$
\begin{array}{r}
{\left[\mathrm{F}_{\left.\mathrm{Lw}, \mathrm{Mx}_{2 \mathrm{n}+1}(\mathrm{x})+\mathrm{F}_{\mathrm{ABw}, \mathrm{Lw}}(\mathrm{x})\right]\left[\mathrm{F}_{\mathrm{Lw}, \mathrm{Mx}}(\mathrm{n}+1\right.}(\mathrm{x})+\mathrm{F}_{\left.\mathrm{STx}_{2 \mathrm{n}+1}, \mathrm{M}_{2 \mathrm{x}+1}(\mathrm{x})\right]}\right.} \\
\geq \quad 4\left[\mathrm{~F}_{\mathrm{ABw}, \mathrm{Lw}}(\mathrm{x} / \mathrm{a})\right]\left[\mathrm{F}_{\left.\mathrm{Mx}_{2 \mathrm{n}+1}, \mathrm{STx}_{2 \mathrm{n}+1}(\mathrm{x})\right] .}\right.
\end{array}
$$

Letting $\mathrm{n} \rightarrow \infty$, we get

$\left[\mathrm{F}_{\mathrm{Lw}, \mathrm{z}}(\mathrm{x})+\mathrm{F}_{\mathrm{z}, \mathrm{Lw}}(\mathrm{x})\right]\left[\mathrm{F}_{\mathrm{Lw}, \mathrm{z}}(\mathrm{x})+\mathrm{F}_{\mathrm{z}, \mathrm{z}}(\mathrm{x})\right] \geq 4\left[\mathrm{~F}_{\mathrm{z}, \mathrm{Lw}}(\mathrm{x} / \mathrm{a})\right]\left[\mathrm{F}_{\mathrm{z}, \mathrm{z}}(\mathrm{x})\right]$,

i.e. $\quad \mathrm{F}_{\mathrm{Lw}, \mathrm{z}}(\mathrm{x}) \geq 1$, yields $\mathrm{Lw}=\mathrm{z}=\mathrm{ABw}$.

Since (L,AB) is compatible and so by proposition (2.4), we have

Hence,

$$
\mathrm{LABw}=\mathrm{ABLw} \text {. }
$$

$$
\mathrm{Lz}=\mathrm{ABz}
$$

Also, $\mathrm{Bz}=\mathrm{z}$ follows from step 4 .

Thus, $\mathrm{Az}=\mathrm{Bz}=\mathrm{Lz}=\mathrm{z}$ and we obtain that $\mathrm{z}$ is the common fixed point of the six maps in this case also.

Step 10. (Uniqueness) Let $\mathrm{u}$ be another common fixed point of $\mathrm{A}, \mathrm{B}, \mathrm{S}, \mathrm{T}, \mathrm{L}$ and $\mathrm{M}$; then $\mathrm{Au}=\mathrm{Bu}=\mathrm{Su}=\mathrm{Tu}=\mathrm{Lu}=\mathrm{Mu}=\mathrm{u}$.

Putting $\mathrm{p}=\mathrm{z}$ and $\mathrm{q}=\mathrm{u}$ for $\mathrm{x}>0$ in (3.1.5), we get

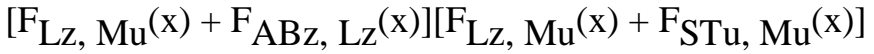

$$
\begin{aligned}
& \geq 4\left[\mathrm{~F}_{\mathrm{ABz}, \mathrm{Lz}}(\mathrm{x} / \mathrm{a})\right]\left[\mathrm{F}_{\mathrm{Mu}}, \mathrm{STu}^{(\mathrm{x})}\right] \text {. }
\end{aligned}
$$

Letting $\mathrm{n} \rightarrow \infty$, we get

$$
\left[\mathrm{F}_{\mathrm{z}, \mathrm{u}}(\mathrm{x})+\mathrm{F}_{\mathrm{z}, \mathrm{z}}(\mathrm{x})\right]\left[\mathrm{F}_{\mathrm{z}, \mathrm{u}}(\mathrm{x})+\mathrm{F}_{\mathrm{u}, \mathrm{u}}(\mathrm{x})\right] \geq 4\left[\mathrm{~F}_{\mathrm{z}, \mathrm{z}}(\mathrm{x} / \mathrm{a})\right]\left[\mathrm{F}_{\mathrm{u}, \mathrm{u}}(\mathrm{x})\right]
$$

i.e. $\quad F_{z, u}(x) \geq 1$, yields $z=u$.

Therefore, $\mathrm{z}$ is a unique common fixed point of $\mathrm{A}, \mathrm{B}, \mathrm{S}, \mathrm{T}, \mathrm{L}$ and $\mathrm{M}$.

This completes the proof. 
V.H.Badshah, Suman Jain and Subhash Mandloi

Remark 3.1. If we take $B=T=I$, the identity map on $X$ in theorem 3.1, then the condition (3.1.2) is satisfied trivially and we get

Corollary 3.1. Let $\mathrm{A}, \mathrm{S}, \mathrm{L}$ and $\mathrm{M}$ be self mappings of a complete Menger space $(\mathrm{X}, \boldsymbol{F}, \mathrm{t})$ satisfying :

(3.1.13) $\mathrm{L}(\mathrm{X}) \subseteq \mathrm{S}(\mathrm{X}), \quad \mathrm{M}(\mathrm{X}) \subseteq \mathrm{A}(\mathrm{X})$;

(3.1.14) Either $\mathrm{A}$ or $\mathrm{L}$ is continuous;

(3.1.15) ( $\mathrm{L}, \mathrm{A})$ is compatible and $(\mathrm{M}, \mathrm{S})$ is semi-compatible;

(3.1.16) for all $\mathrm{p}, \mathrm{q} \in \mathrm{X}, \mathrm{x}>0$ and $0<\alpha<1$,

$$
\begin{gathered}
{\left[\mathrm{F}_{\mathrm{Lp}, \mathrm{Mq}}(\mathrm{x})+\mathrm{F}_{\mathrm{Ap}, \mathrm{Lp}}(\mathrm{x})\right]\left[\mathrm{F}_{\mathrm{Lp}, \mathrm{Mq}}(\mathrm{x})+\mathrm{F}_{\mathrm{Sq}}, \mathrm{Mq}^{(\mathrm{x})}\right]} \\
\geq 4\left[\mathrm{~F}_{\mathrm{Ap}, \mathrm{Lp}}{ }^{(\mathrm{x} / \alpha)]\left[\mathrm{F}_{\mathrm{Mq}}, \mathrm{Sq}^{(\mathrm{x})}\right) .}\right.
\end{gathered}
$$

Then $\mathrm{A}, \mathrm{S}, \mathrm{L}$ and $\mathrm{M}$ have a unique common fixed point in $\mathrm{X}$.

Next we utilize our Theorem 3.1 to prove another common fixed point theorem in a complete metric space.

Theorem 3.2. Let $\mathrm{A}, \mathrm{B}, \mathrm{S}, \mathrm{T}, \mathrm{L}$ and $\mathrm{M}$ be self mappings of a complete metric sapce (X, d) satisfying (3.1.1), (3.1.2), (3.1.3), (3.1.4) and

$$
\begin{array}{r}
{[\mathrm{d}(\mathrm{Lp}, \mathrm{Mq})]^{1 / 2}\left\{[\mathrm{~d}(\mathrm{ABp}, \mathrm{Lp})]^{1 / 2}+[\mathrm{d}(\mathrm{STq}, \mathrm{Mq})]^{1 / 2}\right\}} \\
\leq \alpha\{\mathrm{d}(\mathrm{ABp}, \mathrm{Lp})+\mathrm{d}(\mathrm{Mq}, \mathrm{STq})\},
\end{array}
$$

for all $p, q \in X$ where $0<\alpha<1$.

Then A, B, S, T, L and $\mathrm{M}$ have a unique common fixed point in $\mathrm{X}$.

Proof. The proof follows from theorem 3.1 and by considering the induced Menger space $(X, F, t)$, where $\mathrm{t}(\mathrm{a}, \mathrm{b})=\min \{\mathrm{a}, \mathrm{b}\}$ and $\mathrm{F}_{\mathrm{p}, \mathrm{q}}(\mathrm{x})=\mathrm{H}(\mathrm{x}-\mathrm{d}(\mathrm{p}, \mathrm{q})), \mathrm{H}$ being the distribution function as given in the definition 2.1.

\section{Conclusion}

In view of remark 3.1, corollary 3.1 is a generalization of the result of Singh and Sharma [12] in the sense that the condition of compatibility of the pairs of self maps has been reduced to compatible and semi-compatible self maps and only one of the compatible maps is needed to be continuous.

Acknowledgement. Authors are thankful to the referee for his valuable comments.

\section{REFERENCES}

1. Y.J.Cho, B.K.Sharma and R.D.Sahu, Semi-compatibility and fixed points, Math. Japon., 42 (1) (1995) 91-98.

2. A.Jain and B.Singh, Common fixed point theorem in Menger space through compatible maps of type (A), Chh. J. Sci. Tech., 2 (2005) 1-12.

3. A.Jain and B.Singh, A fixed point theorem in Menger space through compatible maps of type (A), V.J.M.S., 5(2) (2005) 555-568.

4. A.Jain and B.Singh, Common fixed point theorem in Menger Spaces, The Aligarh Bull. of Math., 25(1) (2006) 23-31.

5. K.Jha, M.Imdad and U.Rajopadhyaya, Fixed point theorems for occasionally weakly compatible mappings in semi-metric space, Annals of Pure and Applied Mathematics, 5(2) (2014) 153-157. 
Fixed Point Theorem and Semi-Compatibility in Menger Probabilistic Metric Space

6. K.Jha, R.P.Pant and K.B.Manandhar, A common fixed point theorem for reciprocal continuous compatible mappings in metric space, Annals of Pure and Applied Mathematics, 5(2) (2014) 120-124.

7. K.Menger, Statistical metrics, Proc. Nat. Acad. Sci., 28 (1942) 535 -537.

8. S.N.Mishra, Common fixed points of compatible mappings in PM-spaces, Math. Japon., 36(2) (1991) 283-289.

9. B.Schweizer and A.Sklar, Statistical metric spaces, Pacific J. Math., 10 (1960) 313334.

10. V.M.Sehgal and A.T.Bharucha-Reid, Fixed points of contraction maps on probabilistic metric spaces, Math. System Theory, 6 (1972) 97- 102.

11. B.Singh and S.Jain, Semi-compatibility and fixed point theorem in Menger space, Journal of the Chungcheong Mathematical Society, 17 (1) (2004) 1-17.

12. B.Singh and R.K.Sharma, Common fixed points of compatible maps in Menger spaces, Vikram Mathematical Journal, 16 (1986) 51-56.

13. V.Srinivas and R.Umamaheshwar Rao, Djoudi's common fixed point theorem on compatible mappings of Type (P), Annals of Pure and Applied Mathematics, 6(1) (2014) 19-24. 